\title{
Research on Time-of-use Electricity Price Model Based on Hierarchical Clustering-Price Elasticity Theory
}

\author{
Dong Jun, Wang Pei*, Palidan Ainiwaer, Nie Shilin \\ School of Economics and Management, North China Electric Power University, Beijing, China \\ Email address: \\ dongjun624@126.com (Dong Jun),1608156465@qq.com (Wang Pei),1980989576@qq.com (P. Ainiwaer), \\ 1298940318@qq.com (N. Shilin) \\ ${ }^{*}$ Corresponding author
}

To cite this article:

Dong Jun, Wang Pei, Palidan Ainiwaer, Nie Shilin. Research on Time-of-use Electricity Price Model Based on Hierarchical Clustering-Price Elasticity Theory. American Journal of Electrical Power and Energy Systems. Vol. 8, No. 4, 2019, pp. 95-103.

doi: $10.11648 /$ j.epes.20190804.12

Received: July 10, 2019; Accepted: August 3, 2019; Published: August 15, 2019

\begin{abstract}
The peak-valley time-of-use electricity price can reduce the peak-valley difference of the power system, improve the load factor and operational reliability of the power system, and bring huge economic and social benefits. With the continuous development of society, the resident load will gradually become the main component of the power demand response. Therefore, studying the changes of residential load under the time-of-use electricity price policy is of great significance for the grid companies to better develop demand-side management strategies and carry out load forecasting work. Firstly, this paper combines fuzzy mathematics theory with hierarchical clustering algorithm to divide the peak-to-valley period of the resident load, which ensures the accuracy of the peak-valley period segmentation. Then the load response curve of residents under the condition of time-of-use electricity price is obtained using the electricity demand price elasticity matrix based on the electricity-electricity price elasticity theory. The results show that the time-of-use electricity price policy can motivate users to change their electricity consumption behavior and achieve the effect of peak-cutting and valley filling. The effectiveness of the proposed model and method is verified by numerical simulation. The results show that the time-of-use electricity price policy can motivate users to change their electricity consumption behavior and achieve the effect of peak-cutting and valley filling. The effectiveness of the proposed model and method is verified by numerical simulation.
\end{abstract}

Keywords: Hierarchical Clustering, Price Elasticity Matrix, Time Division, Time-of-use Tariff

\section{Introduction}

With the development of smart grids, demand response has gradually received people's attention. As an important measure of demand side response, time-of-use electricity prices have accumulated rich experience [1-2]. The time-of-use price policy refers to motivating the users to change the power consumption behavior through the price signal to achieve the effect of load transfer and peak-filling, which plays an important role in ensuring the safe operation of the power grid and improving the reliability of the power system [3]. Currently, electric energy has penetrated into all aspects of people's production and life, and has become one of the essential energy sources in life. In addition, with the continuous development of the economy and society and improvement of people's living standard, the wide application of various household appliances has increased the demand for electricity consumption of residents. Therefore, the potential for residents to participate in demand response is huge, which is conducive to increasing the system load rate and increasing the transfer load. Implementing a time-of-use electricity price strategy for residents is of great significance for improving resource utilization and relieving power shortages during peak periods.

At present, many scholars have studied the time-of-use electricity price model. Liu Yan and Zhongfu Tan et al [4] have established a user response model for time-of-use electricity price with reference to the response model of general users, and proposed a method for time-of-use electricity prices based on different time periods. However, they only made a rough division based on other literature's experience in peak-to-valley research, and did not give a specific method of dividing the peak-to-valley period. 
Kischen D S, Strbac G [5-6] et al applied the electricity-electricity price elasticity matrix to comprehensively describe the demand price elasticity to achieve the user's accurate response to the peak-to-valley time-of-use electricity price, which is widely used. Hongchun Shu and Dong Jun [7] proposed the concept of self-elasticity coefficient and cross-elastic coefficient to respectively describe the user response in this period and other periods, but the research is mainly aimed at industrial users, which is more restrictive. Tang Jie, Ren Zhen et al [8] gave a simplified method for obtaining the elastic matrix by analyzing the structural characteristics of the elastic matrix. Zhou Bo, Wang Bo [9] et al adopted the fuzzy membership function and the responsiveness attribute index to divide the peak-to-valley period, and the peak period was divided based on this to enhance the peak clipping effect. Huizhou Liu, Gao Fei et al [10] described two kinds of user response models based on user response curve and demand elasticity, and stepped out the step flow of peak-to-valley time-of-use price model optimization, and analyzed the application scope and improvement trend of the optimization method for time-of-use electricity price model, which is beneficial to the further development of time-of-use (TOU) electricity price. Previous literatures have conducted some research on load curve peak-to-valley period division and user response model, but it is difficult to determine the certain method of dividing the peak-to-valley period, and there are few studies on the residential load curve. Therefore, a method for segmentation of peak-to-valley period of residential residents' load curve is presented in this paper, and the clustering of time points at 96 time points is considered, which has a fine time granularity and enriches the theoretical study of residential load participation in electricity price response.

\section{Theoretical Basis}

\subsection{Fuzzy Membership Theory}

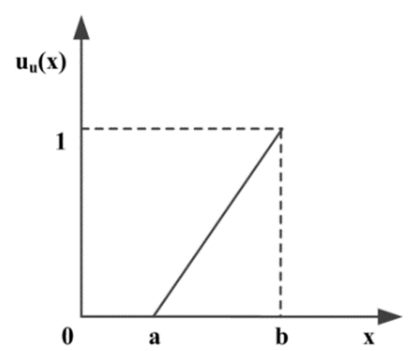

Figure 1. Membership function of partially large semi-trapezoidal.

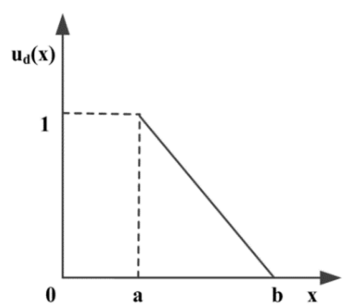

Figure 2. Membership degree function of small and semi-trapezoidal.
The fuzzy membership degree theory belongs to the branch of fuzzy theory which is developed on the basis of the fuzzy set theory established by Professor L. A. zadeh of the Department of Electrical Engineering of the University of California, Berkeley in 1965 to deal with the concept of fuzzy and uncertain things based on quantitative mathematical model. In this paper, the large-scale and partial-small semi-trapezoidal membership functions in fuzzy mathematics are used to determine the fuzzy membership of the residents at 96 time points in the peak, flat and valley periods.

The large and small-semi trapezoidal membership functions respectively represent the peak membership and valley membership at time points. The function image and expression are as follows.

$$
\left\{\begin{array}{l}
u u(x)=\frac{x-a}{b-a} \\
u d(x)=\frac{b-x}{b-a}
\end{array}\right.
$$

In the formula, $x$ represents each time point in 1-24 hours, $u u(x)$ and $u d(x)$ respectively represent the corresponding peak and valley membership degree of each time point, $b$ represents the maximum load at each time point, $a$ represents the minimum load at each time point.

There are several basic principles to consider when dividing the peak-valley period:

(1) The probability that each time point on the load curve is at the peak time and the valley time is determined by the semi-trapezoidal membership function;

(2) The length of each period of peak, flat and valley is generally 5-10 hours;

(3) According to the actual situation of the time division, the general time division is accurate to the hour;

(4) According to empirical theory, a load with peak time membership of not less than $75 \%$ is generally classified as a peak period, and the load with valley time membership of not less than $75 \%$ is classified as the valley period, and load with peak and valley membership between $35 \%$ and $65 \%$ is classified as a flat period, and the load with a peak and valley membership between $65 \%$ and $75 \%$ may belong to a peak period or a flat period.

\subsection{Hierarchical Clustering Theory}

Clustering analysis is a data protocol technology, which can be used to specify a large number of observations into several categories. The main ideas are as follows:

(1) The membership degrees of peaks and valleys at each time point are calculated by using the square Euclidean distance formula. The square Euclidean distance formula is as follows:

$$
d_{i j}=\sum_{t=1}^{m}\left|u_{i t}-u_{j t}\right|^{2}
$$

Among them, $d_{i j}$ denotes the square of the distance 
between membership degree $u_{i k}$ and $u_{j k}$, and $t$ denotes the moment.

(2) According to the distance matrix, n clustering sets are denoted as:

$$
C=\left(\mathrm{c}_{1}, \mathrm{c}_{2}, \mathrm{c}_{3} \cdots c_{n}\right)
$$

The similarity between each cluster tree $\left(\mathrm{c}_{i}, \mathrm{c}_{j}\right)$ in $C$ is as follows:

$$
\operatorname{sim}\left(\mathrm{c}_{i}, \mathrm{c}_{j}\right)=\left(\mathrm{c}_{i} \cdot \mathrm{c}_{j}\right) /\left(\left|\mathrm{c}_{i}\right| \cdot\left|\mathrm{c}_{j}\right|\right)
$$

Among them:

$$
\begin{array}{r}
\left(\mathrm{c}_{i} \cdot \mathrm{c}_{j}\right)=\sum_{t=1}^{n} \mathrm{c}_{i k} \mathrm{c}_{k j} \\
\left|\mathrm{c}_{i}\right|=\sqrt{\sum_{t=1}^{n} \mathrm{c}_{i k} \mathrm{c}_{k j}} \\
\left|\mathrm{c}_{j}\right|=\sqrt{\sum_{t=1}^{n} \mathrm{c}_{j k} \mathrm{c}_{j k}}
\end{array}
$$

(3) The high similarity classes are selected to merge into new classes, and the feature vectors of $\mathrm{c}_{i}$ and $\mathrm{c}_{j}$ are merged to form a new clustering set $C^{\prime}=\left(\mathrm{c}_{1}^{\prime}, \mathrm{c}_{2}^{\prime}, \mathrm{c}_{3}^{\prime} \cdots c_{l}^{\prime}\right)$.

(4) Repeat the above operations until the pre-set conditions are met, and draw the clustering map according to the clustering results.

\subsection{The Theory of Demand-Price Elasticity}

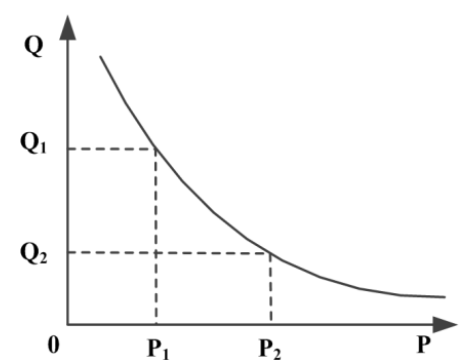

Figure 3. Diagram of the relationship between electricity demand and price.

The term elasticity was first proposed by Alfred Marshall, referring to the sensitivity of one variable to another. In economics, demand price elasticity is generally used to measure the amount of demand as a function of commodity prices. As a special commodity, electricity also obeys the law of electricity consumption changing with price in the market. The variation law of electricity demand with price is shown in Figure 3.

Based on power user psychology, the elasticity coefficient of electricity Quantity-price is defined as follows:

$$
\gamma=\frac{\Delta Q / Q}{\Delta P / P}
$$

Among them,

$$
\begin{gathered}
\Delta Q=Q_{2}-Q_{1} \\
\Delta P=P_{2}-P_{1}
\end{gathered}
$$

Where, $P$ represents electricity price, $\Delta P$ indicates the price change before and after the implementation of the peak-to-valley time-of-use price, $Q$ represents the amount of load, $\Delta Q$ denotes the amount of load change before and after the implementation of peak-to-valley time-of-use price, $P_{1}$ and $P_{2}$ indicates different electricity prices, $Q_{1}$ and $Q_{2}$ denotes the load values at the corresponding electricity prices.

The response form of power users to electricity prices is divided into single-period response and multi-period response. The single-period response means that the user load cannot achieve the transfer of each time period, and the multi-period response can achieve that the user's load shifts from the peak time to the valley time period. In real life, the user's electricity usage behavior is not only related to the price of the current time period, but also affected by the price of other time periods. The self-elastic coefficient and the cross-elastic coefficient of the load and electricity price are obtained by the formula (8).

$$
\left\{\begin{array}{l}
\gamma_{i i}=\frac{\Delta Q_{i} / Q_{i}}{\Delta P_{i} / P_{i}} \\
\gamma_{i j}=\frac{\Delta Q_{i} / Q_{i}}{\Delta P_{j} / P_{j}}
\end{array}\right.
$$

Where, $i$ and $j$ represent different time periods, $Q_{i}$ and $P_{i}$ respectively represent the load and electricity price of the power user before the implementation of the time-of-use price policy in $i$ time periods, $P_{j}$ indicates the electricity price of the user before the implementation of the time-of-use price in $j$ time periods, $\Delta Q_{i}$ and $\Delta P_{i}$ respectively represent the load and price variations of power users before and after the implementation of the time-of-use price in $i$ time periods $\Delta P_{j}$ represent the price variations of power users before and after the implementation of the time-of-use price in $j$ time periods.

The load variations of the power users in each period after the implementation of the peak -valley time-of-use price policy can be expressed as:

$$
\left[\begin{array}{c}
\Delta Q_{1} / Q_{1} \\
\Delta Q_{2} / Q_{2} \\
\Delta Q_{3} / Q_{3} \\
\vdots \\
\Delta Q_{n} / Q_{n}
\end{array}\right]=E\left[\begin{array}{c}
\Delta P_{1} / P_{1} \\
\Delta P_{2} / P_{2} \\
\Delta P_{3} / P_{3} \\
\vdots \\
\Delta P_{n} / P_{n}
\end{array}\right]
$$




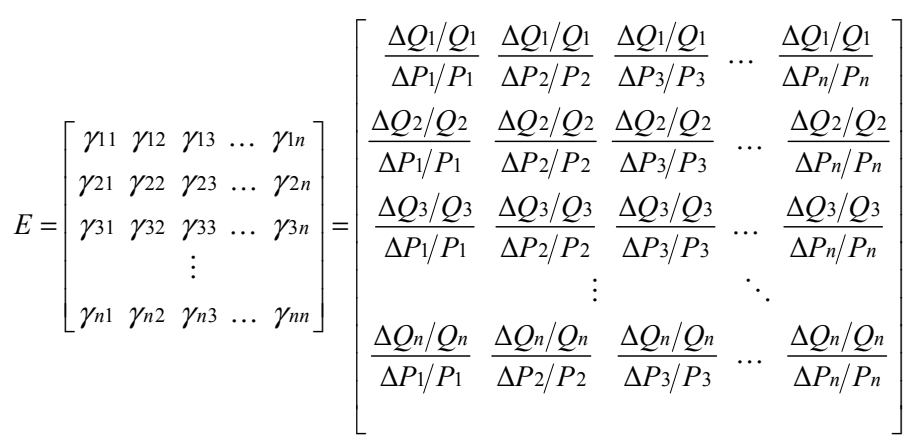

Where, $E$ is the electricity-electricity response elastic matrix of the power user, and $n$ is the number of time slots, so that the load transfer amount after the implementation of the peak-valley time-of-use price is:

$$
\left[\begin{array}{c}
\Delta Q_{1} \\
\Delta Q_{2} \\
\Delta Q_{3} \\
\vdots \\
\Delta Q_{n}
\end{array}\right]=\frac{1}{n}\left[\begin{array}{lllll}
Q_{1} & & & & \\
& Q_{2} & & & \\
& & Q_{3} & & \\
& & & \ddots & \\
& & & & Q_{n}
\end{array}\right] E\left[\begin{array}{c}
\Delta P_{1} / P_{1} \\
\Delta P_{2} / P_{2} \\
\Delta P_{3} / P_{3} \\
\vdots \\
\Delta P_{n} / P_{n}
\end{array}\right]
$$

\section{Hourly Price Response Model for Resident Users}

Based on the basic theory mentioned above, a time-of-use price response model for residential users is constructed. The main processes are as follows:

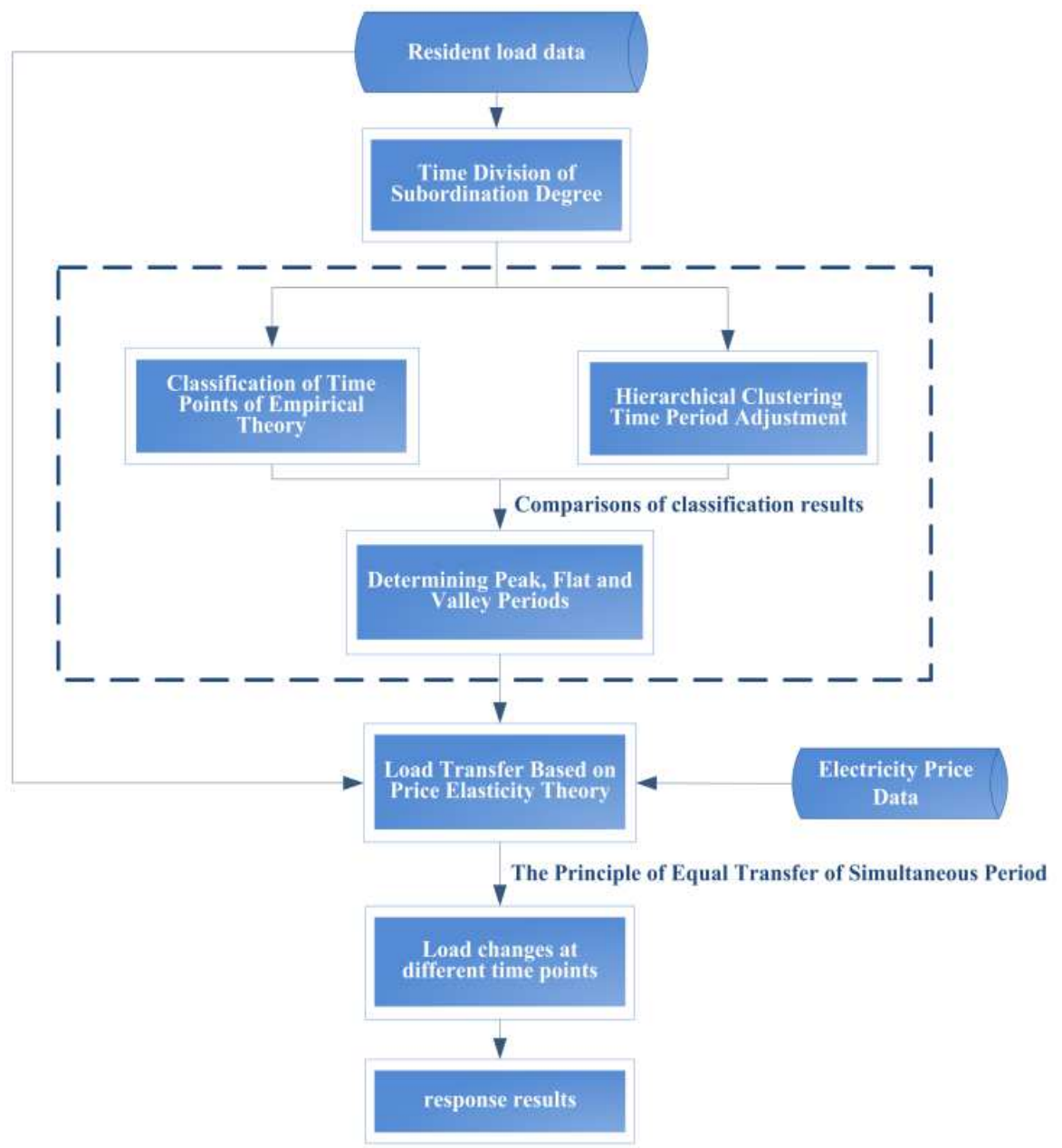

Figure 4. Hourly price response model for resident users.

(1) The original resident load data $Q$ is used to solve out the peak and valley membership degrees at various time points by using the large and small trapezoidal membership functions;

(2) Using empirical theory to classify the obtained peak and 
valley membership degrees;

(3) Based on the basic principle of time division, the hierarchical clustering algorithm is used to re-divide the obtained peak-valley membership degree, and the obtained membership degree of each time point is reclassified according to the empirical theory to obtain the final peak, flat and valley periods: $T_{1} 、 T_{2} 、 T_{3}$;

(4) According to the electric power elasticity matrix, the amount of load transferred in each period and the amount of load at each time point after the response are obtained $Q^{\prime}=\left\{Q_{1}^{\prime}, Q_{2}^{\prime}, Q_{3}^{\prime}, Q_{4}^{\prime} \ldots Q_{96}^{\prime}\right\}$.

\section{Case Study}

The method proposed in this paper is used to analyze the electricity price response of a residential area load, and simulate the change of the residential load curve under the time-of-use electricity price policy. The specific analysis is as follows.

\subsection{Time Division of Time--of-use Electricity Price}

Based on the rule of time-of-use electricity price segmentation, it is generally divided by hourly hour units and uses different electricity prices for power demand side response. In this paper, the load value of the resident's typical day at 96 o'clock is classified into the peak period $T_{1}$, the flat period $T_{2}$, and the valley period $T_{3}$, and it satisfies:

$$
T_{1}+T_{2}+T_{3}=24
$$

The load data of 96 points on the typical day of the residents is selected for analysis in this paper. The load data is shown in Table 1.

Table 1. The load data of 96 points on the typical day.

\begin{tabular}{|c|c|c|c|c|c|c|c|}
\hline time & load/KW & time & load/KW & time & load/KW & time & load/KW \\
\hline $0: 00$ & 277.6 & $6: 00$ & 258.4 & $12: 00$ & 464.4 & $18: 00$ & 686.6 \\
\hline $0: 15$ & 257.1 & $6: 15$ & 203.8 & $12: 15$ & 535.3 & $18: 15$ & 598.4 \\
\hline $0: 30$ & 290.9 & $6: 30$ & 319.2 & $12: 30$ & 465.1 & $18: 30$ & 517.4 \\
\hline $0: 45$ & 303.2 & $6: 45$ & 258.7 & $12: 45$ & 540.2 & $18: 45$ & 609.7 \\
\hline $1: 00$ & 237.1 & $7: 00$ & 288.1 & $13: 00$ & 410.1 & $19: 00$ & 613.4 \\
\hline $1: 15$ & 251.1 & $7: 15$ & 355.7 & $13: 15$ & 455.8 & $19: 15$ & 583.3 \\
\hline $1: 30$ & 255.3 & $7: 30$ & 337.2 & $13: 30$ & 438.3 & $19: 30$ & 640.2 \\
\hline $2: 00$ & 233.1 & $8: 00$ & 452.8 & $14: 00$ & 445.8 & $20: 00$ & 567.1 \\
\hline $2: 15$ & 206.5 & $8: 15$ & 436.1 & $14: 15$ & 401.9 & $20: 15$ & 612.2 \\
\hline $2: 30$ & 239.2 & $8: 30$ & 438.0 & $14: 30$ & 420.3 & $20: 30$ & 587.5 \\
\hline $2: 45$ & 214.6 & $8: 45$ & 474.6 & $14: 45$ & 325.9 & $20: 45$ & 477.7 \\
\hline $3: 00$ & 218.7 & 9:00 & 457.1 & $15: 00$ & 434.7 & $21: 00$ & 557.5 \\
\hline $3: 15$ & 204.9 & $9: 15$ & 464.1 & $15: 15$ & 415.9 & $21: 15$ & 587.1 \\
\hline $3: 30$ & 196.4 & $9: 30$ & 466.3 & $15: 30$ & 403.8 & $21: 30$ & 551.5 \\
\hline 4:00 & 195.1 & $10: 00$ & 506.9 & $16: 00$ & 372.2 & $22: 00$ & 543.6 \\
\hline $4: 15$ & 195.8 & $10: 15$ & 500.3 & $16: 15$ & 394.0 & $22: 15$ & 509.8 \\
\hline $4: 30$ & 200.4 & $10: 30$ & 470.2 & $16: 30$ & 430.8 & $22: 30$ & 455.0 \\
\hline $4: 45$ & 189.8 & $10: 45$ & 422.1 & $16: 45$ & 466.6 & $22: 45$ & 447.4 \\
\hline $5: 00$ & 210.7 & $11: 00$ & 448.8 & $17: 00$ & 480.9 & $23: 00$ & 419.9 \\
\hline $5: 15$ & 224.7 & $11: 15$ & 559.7 & $17: 15$ & 564.4 & $23: 15$ & 310.1 \\
\hline $5: 30$ & 247.3 & $11: 30$ & 570.9 & $17: 30$ & 542.6 & $23: 30$ & 276.7 \\
\hline $5: 45$ & 267.9 & $11: 45$ & 491.6 & $17: 45$ & 588.2 & $23: 45$ & 278.9 \\
\hline
\end{tabular}

Using the fuzzy semi-trapezoidal membership function, the typical daily load data in winter in Table 1 is analyzed, and the peak and valley membership degrees at each time point are obtained. It is shown in Table 2 and Table 3.

Table 2. Typical daily load moment peak membership degree.

\begin{tabular}{|c|c|c|c|c|c|c|c|}
\hline time & peak membership & time & peak membership & time & peak membership & time & peak membership \\
\hline $0: 00$ & $17.67 \%$ & $6: 00$ & $13.81 \%$ & $12: 00$ & $55.27 \%$ & $18: 00$ & $100.00 \%$ \\
\hline $0: 15$ & $13.55 \%$ & $6: 15$ & $2.82 \%$ & $12: 15$ & $69.55 \%$ & $18: 15$ & $82.25 \%$ \\
\hline $0: 30$ & $20.35 \%$ & $6: 30$ & $26.05 \%$ & $12: 30$ & $55.41 \%$ & $18: 30$ & $65.94 \%$ \\
\hline $0: 45$ & $22.83 \%$ & $6: 45$ & $13.87 \%$ & $12: 45$ & $70.53 \%$ & $18: 45$ & $84.52 \%$ \\
\hline $1: 00$ & $9.52 \%$ & $7: 00$ & $19.79 \%$ & $13: 00$ & $44.34 \%$ & $19: 00$ & $85.27 \%$ \\
\hline $1: 15$ & $12.34 \%$ & $7: 15$ & $33.39 \%$ & $13: 15$ & $53.54 \%$ & $19: 15$ & $79.21 \%$ \\
\hline $1: 30$ & $13.18 \%$ & $7: 30$ & $29.67 \%$ & $13: 30$ & $50.02 \%$ & $19: 30$ & $90.66 \%$ \\
\hline $2: 00$ & $8.72 \%$ & $8: 00$ & $52.94 \%$ & $14: 00$ & $51.53 \%$ & $20: 00$ & $75.95 \%$ \\
\hline $2: 15$ & $3.36 \%$ & $8: 15$ & $49.58 \%$ & $14: 15$ & $42.69 \%$ & $20: 15$ & $85.02 \%$ \\
\hline $2: 30$ & $9.94 \%$ & $8: 30$ & $49.96 \%$ & $14: 30$ & $46.40 \%$ & $20: 30$ & $80.05 \%$ \\
\hline $2: 45$ & $4.99 \%$ & $8: 45$ & $57.33 \%$ & $14: 45$ & $27.40 \%$ & $20: 45$ & $57.95 \%$ \\
\hline 3:00 & $5.82 \%$ & $9: 00$ & $53.80 \%$ & $15: 00$ & $49.30 \%$ & 21:00 & $74.01 \%$ \\
\hline
\end{tabular}




\begin{tabular}{llllllll}
\hline time & peak membership & time & peak membership & time & peak membership & time & peak membership \\
\hline $3: 15$ & $3.04 \%$ & $9: 15$ & $55.21 \%$ & $15: 15$ & $45.51 \%$ & $21: 15$ & $79.97 \%$ \\
$3: 30$ & $1.33 \%$ & $9: 30$ & $55.66 \%$ & $15: 30$ & $43.08 \%$ & $21: 30$ & $72.81 \%$ \\
$3: 45$ & $3.58 \%$ & $9: 45$ & $61.78 \%$ & $15: 45$ & $52.66 \%$ & $21: 45$ & $77.84 \%$ \\
$4: 00$ & $1.07 \%$ & $10: 00$ & $63.83 \%$ & $16: 00$ & $36.71 \%$ & $22: 00$ & $71.22 \%$ \\
$4: 15$ & $1.21 \%$ & $10: 15$ & $62.50 \%$ & $16: 15$ & $41.10 \%$ & $22: 15$ & $64.41 \%$ \\
$4: 30$ & $2.13 \%$ & $10: 30$ & $56.44 \%$ & $16: 30$ & $48.51 \%$ & $22: 30$ & $53.38 \%$ \\
$4: 45$ & $0.00 \%$ & $10: 45$ & $46.76 \%$ & $16: 45$ & $55.72 \%$ & $22: 45$ & $51.85 \%$ \\
$5: 00$ & $4.21 \%$ & $11: 00$ & $52.13 \%$ & $17: 00$ & $58.60 \%$ & $23: 00$ & $46.32 \%$ \\
$5: 15$ & $7.02 \%$ & $11: 15$ & $74.46 \%$ & $17: 15$ & $75.40 \%$ & $23: 15$ & $24.21 \%$ \\
$5: 30$ & $11.57 \%$ & $11: 30$ & $76.71 \%$ & $17: 30$ & $71.01 \%$ & $23: 30$ & $17.49 \%$ \\
$5: 45$ & $15.72 \%$ & $11: 45$ & $60.75 \%$ & $17: 45$ & $80.19 \%$ & $23: 45$ & $17.93 \%$ \\
\hline
\end{tabular}

Table 3. Typical daily load moment valley membership degree.

\begin{tabular}{llllllll}
\hline time & $\begin{array}{l}\text { valley } \\
\text { membership }\end{array}$ & time & valley membership & time & $\begin{array}{l}\text { valley } \\
\text { membership }\end{array}$ & time & $\begin{array}{l}\text { valley } \\
\text { membership }\end{array}$ \\
\hline $0: 00$ & $82.33 \%$ & $6: 00$ & $86.19 \%$ & $12: 00$ & $44.73 \%$ & $18: 00$ & $0.00 \%$ \\
$0: 15$ & $86.45 \%$ & $6: 15$ & $97.18 \%$ & $12: 15$ & $30.45 \%$ & $18: 15$ & $17.75 \%$ \\
$0: 30$ & $79.65 \%$ & $6: 30$ & $73.95 \%$ & $12: 30$ & $44.59 \%$ & $18: 30$ & $34.06 \%$ \\
$0: 45$ & $77.17 \%$ & $6: 45$ & $86.13 \%$ & $12: 45$ & $29.47 \%$ & $18: 45$ & $15.48 \%$ \\
$1: 00$ & $90.48 \%$ & $7: 00$ & $80.21 \%$ & $13: 00$ & $55.66 \%$ & $19: 00$ & $14.73 \%$ \\
$1: 15$ & $87.66 \%$ & $7: 15$ & $66.61 \%$ & $13: 15$ & $46.46 \%$ & $19: 15$ & $20.79 \%$ \\
$1: 30$ & $86.82 \%$ & $7: 30$ & $70.33 \%$ & $13: 30$ & $49.98 \%$ & $19: 30$ & $9.34 \%$ \\
$1: 45$ & $87.06 \%$ & $7: 45$ & $61.07 \%$ & $13: 45$ & $52.09 \%$ & $19: 45$ & $4.01 \%$ \\
$2: 00$ & $91.28 \%$ & $8: 00$ & $47.06 \%$ & $14: 00$ & $48.47 \%$ & $20: 00$ & $24.05 \%$ \\
$2: 15$ & $96.64 \%$ & $8: 15$ & $50.42 \%$ & $14: 15$ & $57.31 \%$ & $20: 15$ & $14.98 \%$ \\
$2: 30$ & $90.06 \%$ & $8: 30$ & $50.04 \%$ & $14: 30$ & $53.60 \%$ & $20: 30$ & $19.95 \%$ \\
$2: 45$ & $95.01 \%$ & $8: 45$ & $42.67 \%$ & $14: 45$ & $72.60 \%$ & $20: 45$ & $42.05 \%$ \\
$3: 00$ & $94.18 \%$ & $9: 00$ & $46.20 \%$ & $15: 00$ & $50.70 \%$ & $21: 00$ & $25.99 \%$ \\
$3: 15$ & $96.96 \%$ & $9: 15$ & $44.79 \%$ & $15: 15$ & $54.49 \%$ & $21: 15$ & $20.03 \%$ \\
$3: 30$ & $98.67 \%$ & $9: 30$ & $44.34 \%$ & $15: 30$ & $56.92 \%$ & $21: 30$ & $27.19 \%$ \\
$3: 45$ & $96.42 \%$ & $9: 45$ & $38.22 \%$ & $15: 45$ & $47.34 \%$ & $21: 45$ & $22.16 \%$ \\
$4: 00$ & $98.93 \%$ & $10: 00$ & $36.17 \%$ & $16: 00$ & $63.29 \%$ & $22: 00$ & $28.78 \%$ \\
$4: 15$ & $98.79 \%$ & $10: 15$ & $37.50 \%$ & $16: 15$ & $58.90 \%$ & $22: 15$ & $35.59 \%$ \\
$4: 30$ & $97.87 \%$ & $10: 30$ & $43.56 \%$ & $16: 30$ & $51.49 \%$ & $22: 30$ & $46.62 \%$ \\
$4: 45$ & $100 \% 00$ & $10: 45$ & $53.24 \%$ & $16: 45$ & $44.28 \%$ & $22: 45$ & $48.15 \%$ \\
$5: 00$ & $95.79 \%$ & $11: 00$ & $47.87 \%$ & $17: 00$ & $41.40 \%$ & $23: 00$ & $53.68 \%$ \\
$5: 15$ & $92.98 \%$ & $11: 15$ & $25.54 \%$ & $17: 15$ & $24.60 \%$ & $23: 15$ & $75.79 \%$ \\
$5: 30$ & $88 \% 43 \%$ & $11: 30$ & $23.29 \%$ & $17: 30$ & $28.99 \%$ & $23: 30$ & $82.51 \%$ \\
$5: 45$ & $84.28 \%$ & $11: 45$ & $39.25 \%$ & $17: 45$ & $19.81 \%$ & $23: 45$ & $82.07 \%$ \\
\hline
\end{tabular}

peak membership

valley membership

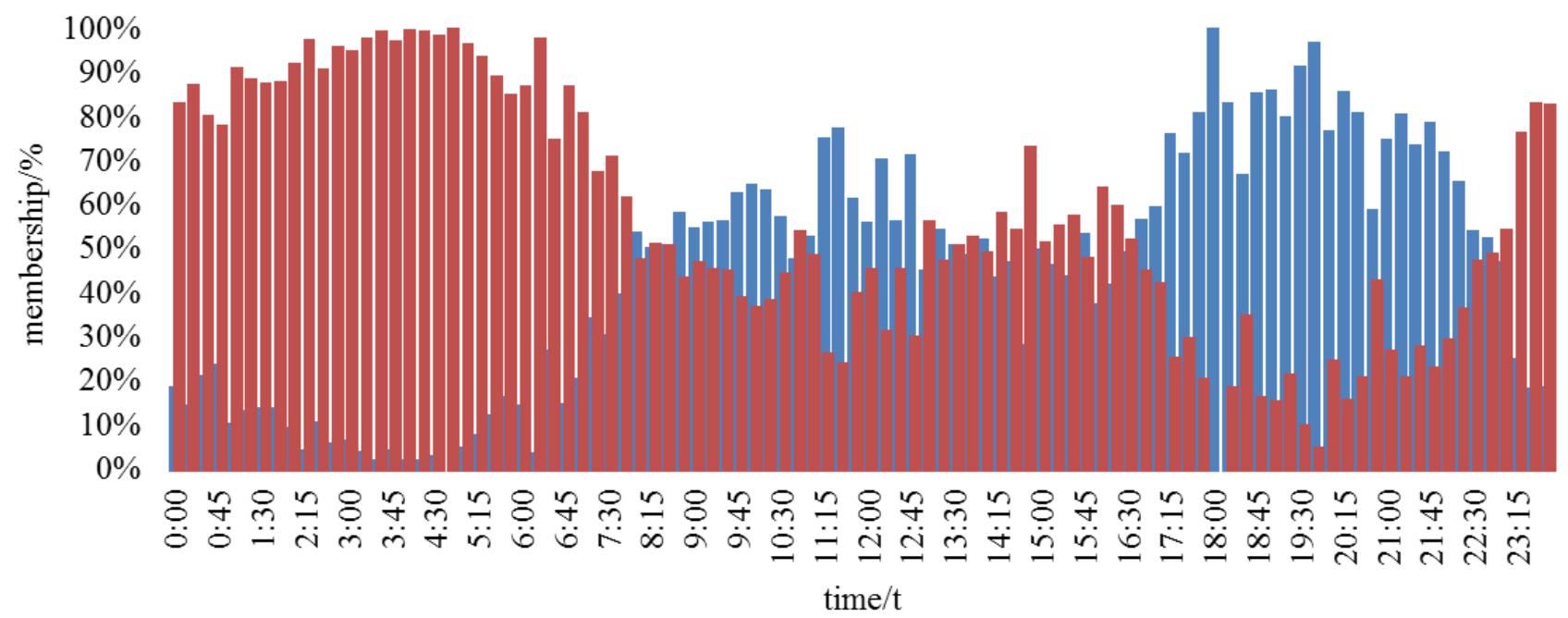

Figure 5. Membership degree of peaks and valleys at each time.

Based on the experience of peak-valley period division, the load with peak period membership of not less than $75 \%$ is classified as the peak period, and the load with the valley period membership of not less than $75 \%$ is classified into the 
valley period, the load with peak and valley membership time between $35 \%$ and $65 \%$ in the peak and valley periods are classified as flat periods, and most of the time points are determined, among which:

$$
\begin{aligned}
& \left\{t_{11: 30}, t_{17: 15}, t_{17: 45}, t_{18: 00}, t_{18: 15}, t_{18: 45}, t_{19: 00}, t_{19: 15}, t_{19: 30}, t_{19: 45},\right. \\
& \left.t_{20: 00}, t_{20: 15}, t_{20: 30}, t_{21: 15}, t_{21: 45}\right\} \subset T_{1} \\
& \left\{t_{7: 45}, t_{8: 00}, t_{8: 15}, t_{8: 30}, t_{8: 45}, t_{9: 00}, t_{9: 15}, t_{9: 30}, t_{9: 45}, t_{10: 00}, t_{10: 15},\right. \\
& t_{10: 30}, t_{10: 45}, t_{11: 00}, t_{11: 45}, t_{12: 00}, t_{12: 30}, t_{13: 00}, t_{13: 15}, t_{13: 30}, t_{13: 45}, \\
& t_{14: 00}, t_{14: 15}, t_{14: 30}, t_{15: 00}, t_{15: 15}, t_{15: 30}, t_{15: 45}, t_{16: 00}, t_{16: 15}, t_{16: 30}, \\
& \left.t_{16: 45}, t_{17: 00}, t_{20: 45}, t_{22: 15}, t_{22: 30}, t_{22: 45}, t_{23: 00}\right\} \subset T_{2} \\
& \left\{t_{0: 00}, t_{0: 15}, t_{0: 30}, t_{0: 45}, t_{1: 00}, t_{1: 15}, t_{1: 30}, t_{1: 45}, t_{2: 00}, t_{2: 15}, t_{2: 30},\right. \\
& t_{2: 45}, t_{3: 00}, t_{3: 15}, t_{3: 30}, t_{3: 45}, t_{4: 00}, t_{4: 15}, t_{4: 30}, t_{4: 45}, t_{5: 00}, t_{5: 15}, \\
& \left.t_{5: 30}, t_{5: 45}, t_{6: 00}, t_{6: 15}, t_{6: 45}, t_{7: 00}, t_{23: 15}, t_{23: 30}, t_{23: 45}\right\} \subset T_{3}
\end{aligned}
$$

In addition, the time $t_{6: 30}, t_{7: 15}, t_{7: 30}, t_{11: 15}, t_{12: 15}, t_{12: 45}$, $t_{14: 45}, t_{17: 30}, t_{18: 30}, t_{21: 00}, t_{21: 30}, t_{22: 00}$ may belong to the peak period or the valley period. Since the peak-to-valley time-of-use electricity price is generally distinguished according to the hour of the hour, the singular points are ignored and the time points that are not classified are merged. The optimized result is:

$$
\begin{gathered}
\left\{t_{17: 15}, t_{17: 30}, t_{17: 45}, t_{18: 00}, t_{18: 15}, t_{18: 30}, t_{18: 45}, t_{19: 00}, t_{19: 15}, t_{19: 30},\right. \\
\left.t_{19: 45}, t_{20: 00}, t_{20: 15}, t_{20: 30}, t_{20: 45}, t_{21: 00} t_{21: 15}, t_{21: 30}, t_{21: 45}\right\} \subset T_{1} \\
\left\{t_{7: 15}, t_{7: 30}, t_{7: 45}, t_{8: 00}, t_{8: 15}, t_{8: 30}, t_{8: 45}, t_{9: 00}, t_{9: 15}, t_{9: 30}, t_{9: 45}, t_{10: 00},\right. \\
t_{10: 15}, t_{10: 30}, t_{10: 45}, t_{11: 00}, t_{11: 15}, t_{11: 30}, t_{11: 45}, t_{12: 00}, t_{12: 15}, t_{12: 30}, t_{12: 45}, \\
t_{13: 00}, t_{13: 15}, t_{13: 30}, t_{13: 45}, t_{14: 00}, t_{14: 15}, t_{14: 30}, t_{14: 45}, t_{15: 00}, t_{15: 15}, t_{15: 30}, \\
\left.t_{15: 45}, t_{16: 00}, t_{16: 15}, t_{16: 30}, t_{16: 45}, t_{17: 00}, t_{22: 15}, t_{22: 30}, t_{22: 45}, t_{23: 00}\right\} \subset \\
T_{2} \\
\left\{t_{0: 00}, t_{0: 15}, t_{0: 30}, t_{0: 45}, t_{1: 00}, t_{1: 15}, t_{1: 30}, t_{1: 45}, t_{2: 00}, t_{2: 15}, t_{2: 30}, t_{2: 45},\right. \\
t_{3: 00}, t_{3: 15}, t_{3: 30}, t_{3: 45}, t_{4: 00}, t_{4: 15}, t_{4: 30}, t_{4: 45}, t_{5: 00}, t_{5: 15}, t_{5: 30}, t_{5: 45}, \\
\left.t_{6: 00}, t_{6: 15}, t_{6: 30}, t_{6: 45}, t_{7: 00}, t_{23: 15}, t_{23: 30}, t_{23: 45}\right\} \subset T_{3}
\end{gathered}
$$

Therefore, the time division interval for the time-of-use price is: peak time: 17:00-22:00; flat time: 7:00-17:00, 22:00-23:00; valley time: 23:00-next day 7:00. In order to make the division of the peak, flat, valley time periods more reasonable, the method of hierarchical clustering is used to verify, and the clustering analysis results of peak and valley membership are carried out by SPSS software, which is depicted in figure 6 .

It can be seen from the cluster analysis that the general method of classifying each time point according to the empirical theory has a reference function, which is basically consistent with the peak, flat and valley periods of the cluster analysis, and further verifies the accuracy of the time division.

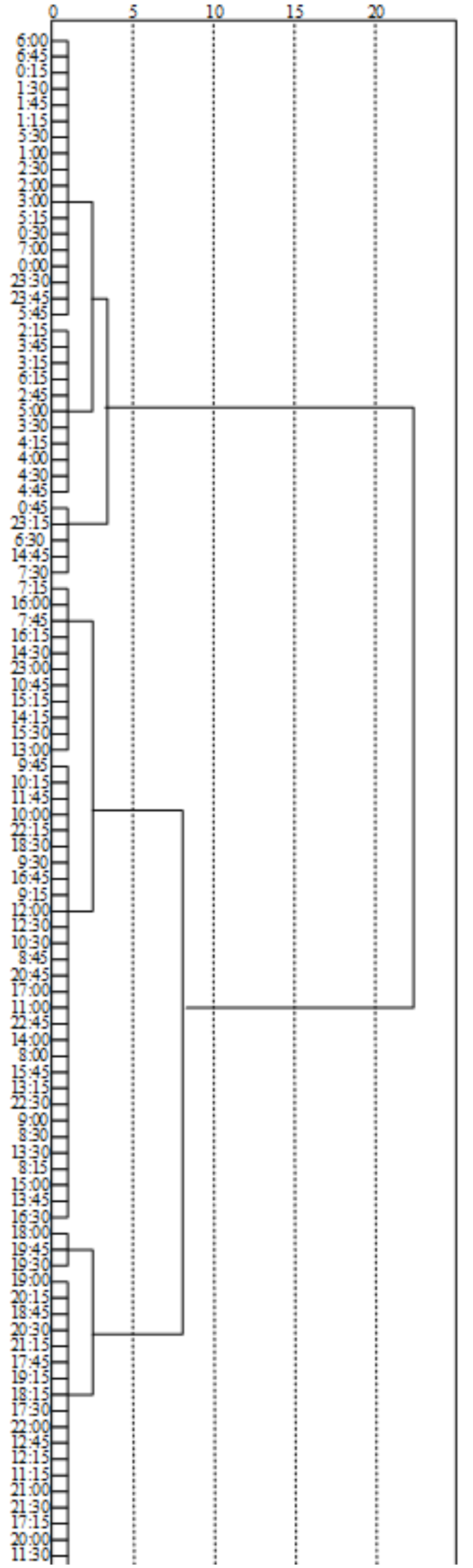

Figure 6. Cluster tree chart of load at each time point in typical days. 


\subsection{Load Transfer}

In calculating the amount of residential load transfer, the following three basic assumptions are assumed:

(1) Before and after the implementation of peak-valley time-of-use electricity prices, the total daily electricity consumption of users remained basically the same or increased.

(2) The transferred electricity in the peak, flat, and valley time periods is evenly distributed on the time axis. The peak clipping or valley filling through the price lever does not affect the shape of the peak-to-valley load curve.

(3) Only consider the impact between electricity price adjustment and load demand. This assumption indicates that the factors affecting the user's demand response law only consider the price of electricity, and the influence of other factors is not considered in this paper.

The existence of the basic assumptions makes the discussion and analysis of this paper greatly simplified and can be carried out smoothly. The load curve is optimized based on the time-of-use period calculated in the previous paper. $Q_{\text {peak }}=11671.6 \mathrm{KW} \quad, \quad Q_{\text {flat }}=19745.6 \mathrm{KW}$,
$Q_{\text {valley }}=7773.0 \mathrm{KW}$ is known. Since the current residential price of a city has only implemented the ladder price and there is no pricing strategy for time-of-use electricity price, it is analyzed and calculated according to the electricity price in literature [11], that is, the peak time price is 0.6557 yuan $/ \mathrm{KWh}$, and the electricity price during the flat time periods is 0.5056 yuan $/ \mathrm{KWh}$, the valley time period price is $0.331 \mathrm{yuan} / \mathrm{KWh}$, and the catalogue electricity price is 0.49 yuan / KWh as the basic electricity price. The elasticity coefficient of demand response price is related to many factors, it is necessary to carry out a large number of investigations and analysis in actual calculation, which is more difficult. Therefore, the power elasticity coefficient of residents in the literature [12] is reasonably selected as the basis for analysis.

Available from formula (14):

$$
\left[\begin{array}{c}
\Delta Q_{\text {peak }} \\
\Delta Q_{\text {flat }} \\
\Delta Q_{\text {valley }}
\end{array}\right]=\left[\begin{array}{c}
-107.622 \\
19.975 \\
39.309
\end{array}\right]
$$

The optimized typical daily load curve is shown in Figure 7.

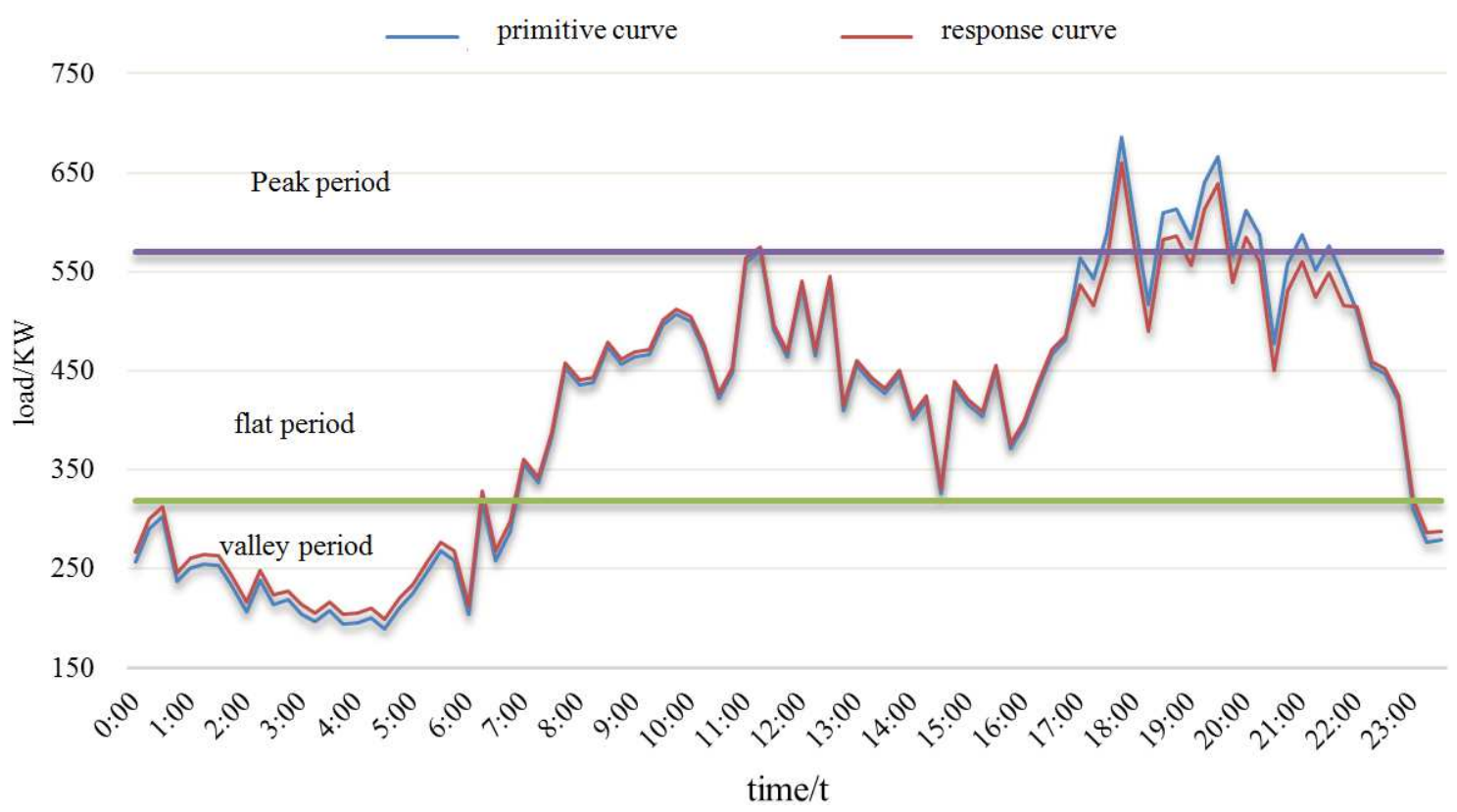

Figure 7. Typical daily load curve before and after adjustment.

It can be seen from the above figure that residents have a response behavior to time-of-use pricing policy, and peak load cutting has a certain potential, mainly transferring the load in peak period to flat period and valley period. Due to the small electricity base of residents, the amount of electricity consumption is related to the residents' income, power-saving consciousness, and the load transfer capacity is limited, relevant departments should strengthen guidance to promote the load transfer of residents in the community.

\section{Conclusion}

The effect of time-of-use electricity price has an important relationship with the division of peak-to-valley time periods, so only a reasonable division of peaks and valleys can achieve satisfactory results. This paper introduces the method of dividing peaks, valleys and flat periods, and further divides the fuzzy membership using the hierarchical membership algorithm based on the classification of fuzzy membership degree according to empirical theory, which proves that the empirical theory has certain reference significance for the time-of-use price division and reflects the accuracy and reliability of the clustering algorithm. Besides, on the basis of time division, the electricity-electricity price elasticity matrix is used to obtain the load response curve of residents under the time-of-use electricity price policy. It can be seen from the 
comparison of the load curve before and after the response that the electricity demand price elasticity matrix model can effectively guide user demand, alleviate the contradiction between power supply and demand, and maintain stable power load, but residents' participation in demand response is limited. With the development of the economy and society, smart household appliances have entered the residents' lives, and the adjustable load has gradually increased, and the resident load has a great potential to participate in the electricity price response.

In this paper, when studying the application of time-of-use electricity price for residents, only one main related factor is considered. However, in order to formulate time divisions and electricity price response methods that are more in line with actual conditions, other factors should be considered comprehensively in actual practice, such as: residential comfort, residential electricity type, working day and weekend.

\section{Funding}

This research was funded by Beijing social science foundation research base project (18JDGLB037).

\section{References}

[1] Wang Beibei, Li Yang, Gao Zhiwei. Demand Side Management Prospect and Consideration in the Framework of Smart Grid [J]. Power System Automation, 2009, 33 (20): 17-22.

[2] STRBAC G. Demand side management: benefits and challenges [J]. Energy Policy, 2008, 36 (12): 4419-4426.
[3] Ruan Wenjun, Wang Beibei, Li Yang, et al. [J]. Research on User Response Behavior under Peak-Valley Time-of-Use Price [J]. Power Grid Technology, 2012, 36 (7): 86-93.

[4] Liu Yan, Tan Zhongfu, Qi Jianxun. Optimal model of TOU tariff design in peak and valley [J]. China Management Science, 2005, 13 (5): 87-92.

[5] Goran Strbac. Demand side management: Benefits and challenges [J]. Energy Policy, 2008, 36 (12): 4419-4426.

[6] Tang Yudong, Song Hongkun, Hu Funian, etal. Inves-tigation on TOU pricing principles [C]. Transmission and Distribution Conference and Exhibition: Dalian: IEEE/PES, 2005: 1-9.

[7] Shu Hongchun, Dong Jun, Wu Shuijun, et al. Preliminary study on the implementation of Time-of-Use tariff in peak and valley in sales-side industries [J]. Power system automation, 2006, 30 (14): 36-40.

[8] Tang Jie, Ren Zhen, Chen Liang, et al. A new peak-valley time-of-use pricing model and its simulation strategy $[\mathrm{J}]$. Power automation equipment, 2006, 26 (8): 1-4.

[9] Zhou Bo, Wang Bo, Gao Song and Dai Rui. Time-sharing tariff model based on user response [J]. Smart grid, 2016, (03): 307-311.

[10] Liu Huizhou, Gaofei, Hu Xiaojian. Study on optimization method of time-sharing price model [J]. DSM, 2013, (04): $11-14+23$

[11] Liu Ming. Study on pricing strategy of residential peak-valley time-of-use electricity price based on data mining [D]. Guizhou University, 2018.

[12] Zhang Liying, Tan Zhongfu, Wang Mianbin, Qi Jianxun, Zhang Rong. Time-sharing tariff hierarchical optimization model considering uncertain response [J]. China Management Science, 2009, 17 (01): 50-57. 